\title{
EXTERIOR ALGEBRA RESOLUTIONS ARISING FROM HOMOGENEOUS BUNDLES
}

\author{
GUNNAR FL $\varnothing$ YSTAD
}

\begin{abstract}
We describe resolutions of general maps (resp. general symmetric and skew-symmetric maps) $E^{a} \rightarrow E(1)^{b}$ given by linear forms over the exterior algebra $E$. Via the BGG-correspondence we describe the associated coherent sheaves. We also show how representation theory of algebraic groups enables one to solve these types of problems for much larger classes of maps.
\end{abstract}

\section{Introduction}

When $R$ is a commutative ring, the minimal free resolution of a map $R^{a} \rightarrow R^{b}$ and symmetric and skew-symmetric maps $R^{a} \rightarrow R^{a}$, under suitable generality conditions, are well known and have been developed by a series of authors. See [2, A2.6] for an overview.

In this note we do an analog for the exterior algebra $E=\oplus \wedge^{i} V$ on a finite dimensional vector space $V$ and general graded maps $E^{a} \rightarrow E(1)^{b}$, and general graded symmetric and skew-symmetric maps $E^{a} \rightarrow E(1)^{a}$. Since $E$ is both a projective and injective $E$-module, by taking a free (projective) and cofree (injective) resolution of such maps, there is associated an unbounded acyclic complex of free $E$-modules, called a Tate resolution, see [3] or [4]. Via the Bernstein-Gel'fand-Gel'fand (BGG) correspondence, this corresponds to a complex of coherent sheaves on the projective space $\mathrm{P}\left(V^{*}\right)$. We show that in all the cases above (with the dimension of $V$ not too low), this complex actually reduces to a coherent sheaf. We describe these coherent sheaves and also describe completely the Tate resolutions.

These descriptions turn out to be simpler to work out than in the corresponding commutative case, and, maybe at first surprising, the descriptions are also more geometric.

In fact not only are we able to describe the Tate resolutions and coherent sheaves associated to the maps stated above, but, using the theory of representations of reductive groups, we are able to describe the Tate resolutions and coherent sheaves associated to vast larger classes of natural maps $E^{a} \rightarrow E(r)^{b}$,

Received March 3, 2003. 
something which would have required considerably more effort for commutative rings.

There is only one catch related to all our descriptions. We must assume that the dimension of $V$ is not too small compared to $a$ and $b$. For instance for a general map $E^{a} \rightarrow E(1)^{b}$ we must assume that the dimension of $V$ is $\geq a+b-1$. In case the dimension of $V$ is smaller than this, the nature of the problem changes, and we do not investigate this case.

\section{Tate resolutions and projections}

Let $V$ be a finite dimensional vector space over a field $k$. Put $W=V^{*}$ and set $v=\operatorname{dim} \mathrm{P}(W)$. Let $E(V)$ be the graded exterior algebra $\oplus_{i=0}^{v+1} \wedge^{i} V$ where $V$ has degree -1 (because we consider $W$ to have degree 1). Let $\omega_{E}=\operatorname{Hom}_{k}(E, k)$ be the graded dual, which we consider as a left $E$-module. (As such $\omega_{E} \cong$ $E(-v-1)$.) It is the injective hull of $k$.

\subsection{Tate resolutions}

A Tate resolution is an (unbounded) acyclic complex $T$ with components

$$
T^{p}=\oplus_{i \in \mathbf{Z}} \omega_{E}(-i) \otimes_{k} V_{i}^{p}
$$

of finite rank. (The $V_{i}^{p}$ are finite dimensional vector spaces.) Note that a Tate resolution is completely determined, up to homotopy, by each differential $d^{p}$ since $T^{\leq p}$ is a projective resolution of the image of $d^{p}$ and $T^{>p}$ is an injective resolution of the image of $d^{p}$.

By [3] or [4], to each coherent sheaf $\mathscr{F}$ on $\mathrm{P}(W)$ there is associated a Tate resolution $T(\mathscr{F})$ whose terms are

$$
T(\mathscr{F})^{p}=\oplus_{i=0}^{v} \omega_{E}(i-p) \otimes_{k} H^{i} \mathscr{F}(p-i) .
$$

(In particular we see that $T(\mathscr{F})^{p}$ is $\omega_{E}(-p) \otimes_{k} H^{0} \mathscr{F}(p)$ for $p \gg 0$.) The maps

$$
\omega_{E}(i-p) \otimes_{k} H^{i} \mathscr{F}(p-i) \longrightarrow \omega_{E}(i-p-1) \otimes_{k} H^{i} \mathscr{F}(p+1-i)
$$

are determined by the maps in degree $p+1-i$ which are the natural maps

$$
W \otimes_{k} H^{i} \mathscr{F}(p-i) \longrightarrow H^{i} \mathscr{F}(p+1-i) .
$$

Conversely, given a left module $N=\oplus_{i \in \mathbf{Z}} N_{i}$ over $E$ we get associated a complex of coherent sheaves

$$
L(N)^{\sim}: \cdots \rightarrow \mathscr{O}_{\mathrm{P}(U)}(i) \otimes_{k} N_{i} \rightarrow \mathcal{O}_{\mathrm{P}(U)}(i+1) \otimes_{k} N_{i+1} \rightarrow \cdots .
$$


In this way we can to each Tate resolution $T$ associate a complex of coherent sheaves on $\mathrm{P}(W)$ by using this construction on $\operatorname{ker} d^{p}$. (All the $L\left(\operatorname{ker} d^{p}\right)^{\sim}$ become isomorphic in the derived category of coherent sheaves on $\mathrm{P}(W)$. If $T=T(\mathscr{F})$ then $L\left(\operatorname{ker} d^{p}\right)^{\sim}$ is a complex isomorphic to $\mathscr{F}$ in this derived category.)

Remark 1.1. If $K^{\circ}(E-c F)$ is the homotopy category of Tate resolutions, and $D^{b}(\operatorname{coh} / \mathrm{P}(W))$ is the derived category of coherent sheaves on $\mathrm{P}(W)$, the Bernstein-Gel'fand-Gel'fand correspondence says that there is an equivalence of categories

$$
K^{\circ}(E-c F) \cong D^{b}(\operatorname{coh} / \mathrm{P}(W)) .
$$

See [4] or originally [1].

\subsection{Projections}

Let $U \subseteq W$ be a subspace and let $E\left(U^{*}\right)=\oplus \wedge^{i} U^{*}$. The center of the projection $\pi: \mathrm{P}(W) \rightarrow \mathrm{P}(U)$ is the linear subspace $\mathrm{P}(W / U)$. Suppose the support of $\mathscr{F}$ is disjoint from $\mathrm{P}(W / U)$. According to [4],

$$
\operatorname{Hom}_{E(V)}\left(E\left(U^{*}\right), T(\mathscr{F})\right)
$$

is also a Tate resolution (for $E\left(U^{*}\right)$ ) and it is the Tate resolution associated to $\pi_{*} \mathscr{F}$. Note that this gives

$$
T\left(\pi_{*} \mathscr{F}\right)^{p}=\oplus_{i=0}^{\operatorname{dim} \mathrm{P}(U)} \omega_{E\left(U^{*}\right)}(i-p) \otimes_{k} H^{i} \mathscr{F}(p-i) .
$$

If $Y \rightarrow W$ is a linear map, then let $U$ be the image in $W$. Thus we get a projection and an embedding

$$
\mathrm{P}(W) \stackrel{-\rightarrow}{ }(U) \stackrel{i}{\longrightarrow} \mathrm{P}(Y) .
$$

In this case

$$
\operatorname{Hom}_{E(V)}\left(E\left(Y^{*}\right), T(\mathscr{F})\right)
$$

is the Tate resolution of $i_{*} \pi_{*} \mathscr{F}$.

\section{Tate resolutions arising from general maps}

Let $A$ and $B$ be finite dimensional vector spaces. Fix a basis $x_{0}, \ldots, x_{a}$ of $A^{*}$ and $y_{0}, \ldots, y_{b}$ of $B^{*}$ Then a map

$$
A^{*} \longrightarrow V \otimes B
$$


corresponds to a $(b+1) \times(a+1)$ matrix $\left[v_{i j}\right]$ where the $v_{i j}$ are in $V$. It comes from the generic map

$$
A^{*} \longrightarrow\left(A^{*} \otimes B^{*}\right) \otimes B
$$

corresponding to the generic $(b+1) \times(a+1)$ matrix $\left[x_{j} y_{i}\right]$, composed with a map $A^{*} \otimes B^{*} \rightarrow V$ sending $x_{j} y_{i}$ to $v_{i j}$. We shall assume this map is general and it gives us a general morphism

$$
\omega_{E} \otimes A^{*} \longrightarrow \omega_{E}(-1) \otimes B
$$

which again gives a Tate resolution by taking projective and injective resolutions. This again is associated to a complex of coherent sheaves which we now show is a coherent sheaf. Note that $a$ and $b$ are the dimensions of $\mathrm{P}(A)$ and $\mathrm{P}(B)$.

THEOREM 2.1. Given a general morphism

$$
\omega_{E}(a) \otimes A^{*} \stackrel{d}{\longrightarrow} \omega_{E}(a-1) \otimes B
$$

coming from a general surjection $A^{*} \otimes B^{*} \rightarrow V$ and suppose $v \geq a+b$. Let $T$ be the associated Tate resolution with (1) in components 0 and 1 .

a. $T$ is the Tate resolution associated to $\pi_{*} \mathscr{L}$ where $\mathscr{L}$ is the line bundle $\mathcal{O}_{\mathrm{P}(A) \times \mathrm{P}(B)}(-2, a) \otimes \wedge^{a+1} A$ on the Segre embedding of $\mathrm{P}(A) \times \mathrm{P}(B)$ in $\mathrm{P}(A \otimes$ $B)$ and $\pi: \mathrm{P}(A \otimes B) \rightarrow \mathrm{P}(W)$ is the projection.

$b$. The Tate resolution has terms

$$
T^{p}= \begin{cases}\omega_{E}(-p) \otimes S^{p-2} A \otimes S^{p+a} B \otimes \wedge^{a+1} A, & p \geq 2, \\ \omega_{E}(a+b+r) \otimes S^{b+r+1} A^{*} \otimes S^{r-1} B^{*} \otimes \wedge^{b+1} B^{*}, & p=-r<0\end{cases}
$$

Proof. We find

$$
\begin{aligned}
H^{a} \mathscr{L}(-a) & =\wedge^{a+1} A \otimes H^{a} \mathcal{O}_{\mathrm{P}(A) \times \mathrm{P}(B)}(-2-a, 0) \\
= & \wedge^{a+1} A \otimes A^{*} \otimes \wedge^{a+1} A^{*}=A^{*} \\
H^{a} \mathscr{L}(-a+1) & =\wedge^{a+1} A \otimes H^{a} \mathscr{O}_{\mathrm{P}(A) \times \mathrm{P}(B)}(-1-a, 1) \\
& =\wedge^{a+1} A \otimes \wedge^{a+1} A^{*} \otimes B=B
\end{aligned}
$$

and also $H^{i} \mathscr{L}(-i)$ and $H^{i} \mathscr{L}(-i+1)$ are zero for $i \neq a$. The map

$$
(A \otimes B) \otimes H^{a} \mathscr{L}(-a) \rightarrow H^{a} \mathscr{L}(-a+1)
$$


is clearly the generic one and hence we get part a. Part b. follows of course by considering the cohomology of $\mathscr{L}$.

REMARK 2.2. Consider the generic morphism

$$
\mathcal{O}_{\mathrm{P}(A \otimes B)} \otimes A^{*} \rightarrow \mathcal{O}_{\mathrm{P}(A \otimes B)}(1) \otimes B .
$$

The rank strata of this morphism are the orbits in $\mathrm{P}(A \otimes B)$ of $\mathrm{GL}(A) \times \mathrm{GL}(B)$. The stratum of lowest rank one, corresponds to the closed orbit of $\operatorname{GL}(A) \times$ $\mathrm{GL}(B)$ which is the Segre embedding $\mathrm{P}(A) \times \mathrm{P}(B) \hookrightarrow \mathrm{P}(A \otimes B)$.

\section{The Tate resolution of a general symmetric map}

Given now a symmetric map

$$
A^{*} \longrightarrow V \otimes A .
$$

It corresponds to a symmetric matrix $\left[v_{i j}\right]$ of size $a+1$, i.e. with $v_{i j}=v_{j i}$, and comes from the natural map

$$
A^{*} \rightarrow S^{2} A^{*} \otimes A,
$$

corresponding to the generic symmetric matrix $\left[x_{i} x_{j}\right]$, composed with the map $S^{2} A^{*} \rightarrow V$ sending $x_{i} x_{j}$ to $v_{i j}$. We then get a symmetric morphism

$$
\omega_{E} \otimes A^{*} \rightarrow \omega_{E}(-1) \otimes A .
$$

THEOREM 3.1. Given a general symmetric morphism

$$
\omega_{E}(a) \otimes A^{*} \stackrel{d}{\longrightarrow} \omega_{E}(a-1) \otimes A
$$

coming from a general surjection $S^{2} A^{*} \rightarrow V$ with $v \geq 2 a$. Let $T$ be the associated Tate resolution with (2) as the components in degree 0 and 1.

a. $T$ is the Tate resolution associated to $\pi_{*} \mathscr{L}$ where $\mathscr{L}$ is the line bundle $\mathcal{O}_{\mathrm{P}(A) \times \mathrm{P}(A)}(-2, a) \otimes \wedge^{a+1} A$ on the Segre embedding of $\mathrm{P}(A) \times \mathrm{P}(A)$ in $\mathrm{P}(A \otimes A)$ and $\pi: \mathrm{P}(A \otimes A) \rightarrow \mathrm{P}\left(S^{2} A\right) \rightarrow \mathrm{P}(W)$ is the projection.

$b$. The Tate resolution has terms

$$
T^{p}= \begin{cases}\omega_{E}(-p) \otimes S^{p-2} A \otimes S^{p+a} A \otimes \wedge^{a+1} A, & p \geq 2, \\ \omega_{E}(2 a+r) \otimes S^{a+r+1} A^{*} \otimes S^{r-1} A^{*} \otimes \wedge^{a+1} A^{*}, & p=-r<0\end{cases}
$$

Proof. This follows from the previous theorem by verifying that the Segre embedding $\mathrm{P}(A) \times \mathrm{P}(A) \hookrightarrow \mathrm{P}(A \otimes A)$ does not intersect the center of the projection $\mathrm{P}(A \otimes A) \rightarrow \mathrm{P}\left(S^{2} A\right)$. 
In fact the center is the linear subspace $\mathrm{P}\left(\wedge^{2} A\right)$ in $\mathrm{P}(A \otimes A)$. It consists of all points with coordinates given by non-zero skewsymmetric matrices $\left[k_{i j}\right]$, i.e. $k_{i j}=-k_{j i}$. On the other hand the image of the Segre embedding in $\mathrm{P}(A \otimes A)$ are all points given by coordinates $\left[k_{i} l_{j}\right]$ where $\left(k_{0}, \ldots, k_{a}\right)$ and $\left(l_{0}, \ldots, l_{a}\right)$ are points in $\mathrm{P}(A)$ and $\mathrm{P}(A)$. It is easily seen that these sets of points are disjoint.

We now wish to describe the Tate resolution and coherent sheaf associated to a general skew-symmetric morphism. From the preceding one might think that this is quite analogous to what we have done for symmetric maps. However it is quite different due to the fact that the projection center of $\mathrm{P}(A \otimes A) \rightarrow$ $\mathrm{P}\left(\wedge^{2} A\right)$, which is $\mathrm{P}\left(S^{2} A\right)$, intersects the Segre embedding $\mathrm{P}(A) \times \mathrm{P}(A) \hookrightarrow$ $\mathrm{P}(A \otimes A)$. In fact the image of the diagonal are the points with coordinates $\left[k_{i} k_{j}\right]$ and this is a symmetric matrix.

We must therefore take another approach which will give us a much more powerful understanding of what we have just done.

\section{Homogeneous bundles on homogeneous spaces}

We present here some facts about induced homogeneous bundles on homogeneous varieties. In particular we consider their cohomology. We base ourselves on [5].

Let $G$ be a reductive algebraic group over a field $k$ of characteristic zero, with Borel group $B$ and maximal torus $T$. We let $X(T)$ be the characters of $T$ and $R$ the root system of $G$. Corresponding to the choice of $B$ there is a positive root system $R^{+}$and let $S$ be the associated simple roots.

Given a parabolic subgroup $P$ of $G$, we let $P$-mod be the category of rational representations of $P$. For each $P$-module $M$ we get a $G$-equivariant locally free sheaf $\mathscr{L}(M)$ of rank $\operatorname{dim}_{k} M$ on the homogeneous variety $G / P$ [5, I.5.8]. If $N$ is another $P$-module, then $\mathscr{L}\left(M \otimes_{k} N\right)=\mathscr{L}(M) \otimes_{\mathcal{O}_{G / P}} \mathscr{L}(N)$.

To such a parabolic $P$ is associated a subset $I \subseteq S$ and the characters $X(P)$ are all $\mu \in X(T)$ such that $\langle\mu, \check{\alpha}\rangle=0$ for all $\alpha \in I$. For such $\mu$ we get line bundles $\mathscr{L}(\mu)$ on $G / P$. By [5, II.4.6], $\mathscr{L}(\mu)$ is ample (in fact very ample) on $G / P$ if $\langle\mu, \check{\alpha}\rangle>0$ for all $\alpha \notin I$. Thus we get an embedding

$$
G / P \hookrightarrow \mathrm{P}\left(H^{0}(G / P, \mathscr{L}(\mu))\right)
$$

with $\mathscr{O}_{G / P}(1)=\mathscr{L}(\mu)$.

Let

$$
\operatorname{ind}_{P}^{G}: P-\bmod \rightarrow G-\bmod
$$

be the induction functor [5, I.3.3] and let $R^{n}$ ind $_{P}^{G}$ be its higher derived functors. Then $H^{n}(G / P, \mathscr{L}(M))=R^{n}$ ind $_{P}^{G} M$ by [5, I.5.10]. If $\lambda \in X(T)$, to simplify notation, let $R^{n} \operatorname{ind}_{B}^{G} \lambda=H^{n}(\lambda)$. This is an irreducible $G$-module whose 
character is determined by the Borel-Bott-Weil theorem [5, II.5.5] and the Weyl character formula [5, II.5.10].

Associated to the inclusions $B \subseteq P \subseteq G$ there is by [5, I.4.5] a spectral sequence

$$
E_{2}^{n, m}=R^{n} \operatorname{ind}_{P}^{G} \circ R^{m} \operatorname{ind}_{B}^{P} N \Rightarrow R^{n+m} \operatorname{ind}_{B}^{G} N .
$$

If now $\lambda \in X(T)$ is such that $\langle\lambda, \check{\alpha}\rangle \geq 0$ for all $\alpha$ in $I$, then $R^{m} \operatorname{ind}_{B}^{P} \lambda=0$ for $m>0$ by Kempf vanishing [5, II.4.5]. In this case we therefore get by (3) that

$$
\begin{aligned}
H^{n}(\lambda) & =R^{n} \operatorname{ind}_{P}^{G}\left(\operatorname{ind}_{B}^{P} \lambda\right) \\
& =H^{n}\left(G / P, \mathscr{L}\left(\operatorname{ind}_{B}^{P} \lambda\right)\right) .
\end{aligned}
$$

Considering the $G$-equivariant induced sheaf $\mathscr{L}\left(\operatorname{ind}_{B}^{P} \lambda\right)$ on $G / P \hookrightarrow$ $\mathrm{P}\left(H^{0}(\mu)\right)$ we are thus able to determine all the cohomology groups

$$
\begin{aligned}
H^{n}\left(G / P, \mathscr{L}\left(\operatorname{ind}_{B}^{P} \lambda\right)(r)\right) & =H^{n}\left(G / P, \mathscr{L}\left(\operatorname{ind}_{B}^{P} \lambda\right) \otimes \mathscr{L}(r \mu)\right) \\
& =H^{n}\left(G / P, \mathscr{L}\left(\operatorname{ind}_{B}^{P}(\lambda+r \mu)\right)\right)=H^{n}(\lambda+r \mu) .
\end{aligned}
$$

In particular, for the induced homogeneous bundle $\mathscr{L}=\mathscr{L}\left(\operatorname{ind}_{B}^{P} \lambda\right)$ on $\mathrm{P}\left(H^{0}(\mu)\right)$, the terms of the Tate resolution $T$ of $\mathscr{L}$

$$
T^{p}=\oplus_{i=0}^{\operatorname{dim} G / P} \omega_{E}(i-p) \otimes H^{i} \mathscr{L}(p-i)
$$

may be determined.

Example 4.1. Let $G=\mathrm{GL}(W)$ and let $e_{0}, \ldots, e_{v}$ be a basis for $W$. If $T$ is the diagonal matrices we let $\epsilon_{i}$ be the character sending $\operatorname{diag}\left(t_{0}, \ldots, t_{v}\right)$ to $t_{i}$. We let $B$ be the lower triangular matrices. The positive roots are then $\epsilon_{i}-\epsilon_{j}$ where $i<j$ and the positive simple roots are $\alpha_{i}=\epsilon_{i-1}-\epsilon_{i}$ for $i=1 \ldots v$. Let $P$ be the parabolic subgroup corresponding to $\alpha_{2}, \ldots, \alpha_{v}$, i.e. $P$ consists of all matrices

$$
\left(\begin{array}{cccc}
* & 0 & \cdots & 0 \\
* & * & \cdots & * \\
\vdots & & & \\
* & * & \ldots & *
\end{array}\right) .
$$

Letting $U=\left(e_{1}, \ldots, e_{v}\right)$ there is an exact sequence of $P$-modules

$$
0 \rightarrow U \rightarrow W \rightarrow\left(e_{0}\right) \rightarrow 0 .
$$

Here $U$ is the irreducible representation of $P$ with highest weight $\epsilon_{1}$ and thus $U=\operatorname{ind}_{B}^{P} \epsilon_{1}$. On $G / P=\mathrm{P}(W)$ there is an exact sequence

$$
0 \rightarrow \mathscr{L}(U) \rightarrow \mathscr{L}(W) \rightarrow \mathscr{L}\left(\epsilon_{0}\right) \rightarrow 0 .
$$


Since the Picard group of $G / P$ is generated by $\mathscr{L}\left(\epsilon_{0}\right)$ we have $\mathscr{L}\left(\epsilon_{0}\right)=$ $\mathcal{O}_{\mathrm{P}(U)}(1)$. Since $W$ is a $G$-module, $\mathscr{L}(W)=\mathscr{O}_{\mathrm{P}(U)} \otimes_{k} W$. Thus $\mathscr{L}(U)=$ $\Omega_{\mathrm{P}(W)}(1)$.

Given now a partition

$$
\text { i : } i_{1} \geq i_{2} \geq \cdots \geq i_{v}
$$

where $i_{j}$ are integers. The Schur bundle $S_{\mathbf{i}}\left(\Omega_{\mathrm{P}(W)}(1)\right)$ is then the induced bundle $\mathscr{L}\left(S_{\mathrm{i}} U\right)$ where $S_{\mathrm{i}}$ is the Schur functor, [2, A2.5]. Since $S_{\mathrm{i}} U$ is an irreducible representation with highest weight $i_{1} \epsilon_{1}+\cdots+i_{v} \epsilon_{v}$ we get $S_{\mathrm{i}} U=$ $\operatorname{ind}_{B}^{P}\left(\sum_{j=1}^{n} i_{j} \epsilon_{j}\right)$. It then follows that

$$
\begin{aligned}
H^{r} S_{\mathrm{i}}\left(\Omega_{\mathrm{P}(W)}(1)\right)(p-r) & =H^{r}\left(\mathrm{P}(W), \mathscr{L}\left(S_{\mathrm{i}} U\right) \otimes \mathscr{L}\left((p-r) \epsilon_{0}\right)\right) \\
& =H^{r}\left(G / P, \mathscr{L}\left(\operatorname{ind}_{B}^{P}\left((p-r) \epsilon_{0}+\sum_{j=1}^{n} i_{j} \epsilon_{j}\right)\right)\right) \\
& =H^{r}\left((p-r) \epsilon_{0}+\sum_{j=1}^{n} i_{j} \epsilon_{j}\right) .
\end{aligned}
$$

By the Borel-Bott-Weil theorem this can be calculated and the answer turns out to be the following.

Let $h=h(p)$ be such that $i_{h}>p \geq i_{h+1}$ and let $\mathbf{i}(p)$ be the partition

$$
i_{1}-1 \geq \cdots \geq i_{h}-1 \geq p \geq i_{h+1} \geq \cdots \geq i_{v} .
$$

It then follows that

$$
H^{r}\left((p-r) \epsilon_{0}+\sum_{j=1}^{n} i_{j} \epsilon_{j}\right)=\left\{\begin{array}{ll}
H^{0}\left(\sum_{i=0}^{v} \mathbf{i}(p)_{j} \epsilon_{j}\right), & r=h(p) \\
0, & r \neq h(p)
\end{array} .\right.
$$

In particular the Tate resolution $T$ of $S_{\mathbf{i}}\left(\Omega_{\mathrm{P}(W)}(1)\right)$ has "pure" terms

$$
T^{p}=\omega_{E}(h(p)-p) \otimes S_{\mathbf{i}(p)} W .
$$

REMARK 4.2. More generally one can show that if $\mu=\omega_{\alpha}$ is a fundamental weight corresponding to a short root and $P_{S-\{\alpha\}}$ is the corresponding parabolic group, then all the induced bundles $\mathscr{L}\left(\operatorname{ind}_{B}^{P_{S-\{\alpha\}}} \lambda\right)$ have "pure" Tate resolutions in the above sense.

\section{Tate resolutions arising from general skew-symmetric maps}

Suppose given a skew-symmetric map

$$
A^{*} \rightarrow V \otimes A .
$$


Such a map comes from the natural map

$$
A^{*} \rightarrow \wedge^{2} A^{*} \otimes A
$$

and a map $\wedge^{2} A^{*} \rightarrow V$. It gives rise to a skew-symmetric morphism

$$
\omega_{E} \otimes A^{*} \rightarrow \omega_{E}(-1) \otimes A .
$$

To describe the Tate resolution and associated coherent sheaf on $\mathrm{P}(W)$ consider the Plücker embedding of the Grassmann of lines in $\mathrm{P}(A)$

$$
G(A, 2) \hookrightarrow \mathrm{P}\left(\wedge^{2} A\right) .
$$

Let

$$
0 \rightarrow \mathscr{R} \rightarrow \mathscr{O}_{G} \otimes A \rightarrow \mathscr{E} \rightarrow 0
$$

be the tautological sequence (where the rank of $\mathscr{E}$ is two).

THEOREM 5.1. Given a general skew-symmetric morphism

$$
\omega_{E}(a-1) \otimes A^{*} \rightarrow \omega_{E}(a-2) \otimes A
$$

arising from a general surjection $\wedge^{2} A^{*} \rightarrow V$ and suppose $v \geq 2(a-1)$. Let $T$ be the associated Tate resolution with components in degree 0 and 1 given by (4).

a. $T$ corresponds to the coherent sheaf $\pi_{*} \mathscr{L}$ where $\mathscr{L}$ is the bundle $\left(S^{a} \mathscr{E}\right)$ $(-2) \otimes \wedge^{a+1} A$ on $G(A, 2) \hookrightarrow \mathrm{P}\left(\wedge^{2} A\right)$ and $\pi: \mathrm{P}\left(\wedge^{2} A\right) \rightarrow \mathrm{P}(W)$ is the projection.

b. The components of $T$ are given by

$$
T^{p}=\left\{\begin{array}{ll}
\omega_{E}(-p) \otimes S_{a+p-2, p-2} A \otimes \wedge^{a+1} A, & p \geq 2 \\
\omega_{E}(2 a+r-2) \otimes S_{a+r-1, r-1} A^{*} \otimes \wedge^{a+1} A^{*}, & p=-r<0
\end{array} .\right.
$$

Proof. Choose a basis $e_{0}, \ldots, e_{a}$ for $A$ and let $B$ be the Borel subgroup of lower triangular matrices in GL $(A)$. Let $\left(e_{i}\right)$ be the weight space of $\epsilon_{i}$. Then $G(A, 2)=\mathrm{GL}(A) / P$ where $P$ is the maximal parabolic subgroup associated to the roots $\alpha_{i}=\epsilon_{i-1}-\epsilon_{i}, i \neq 2$, i.e. $P$ consists of matrices

$$
\left(\begin{array}{ccccc}
* & * & 0 & \cdots & 0 \\
* & * & 0 & \cdots & 0 \\
* & * & * & \cdots & * \\
\vdots & & & & \\
* & * & * & \cdots & *
\end{array}\right) .
$$


Note that $\operatorname{dim} G(A, 2)=2(a-1)$ and that by the Plücker embedding $G(A, 2) \hookrightarrow \mathrm{P}\left(\wedge^{2} A\right)$ the canonical bundle $\mathscr{O}_{G}(1)$ is $\mathscr{L}\left(\epsilon_{0}+\epsilon_{1}\right)$, where $\epsilon_{0}+\epsilon_{1}$ is the highest weight of $\wedge^{2} A$.

There is a sequence of $P$-modules

$$
0 \rightarrow\left(e_{2}, \ldots, e_{a}\right) \rightarrow A \rightarrow\left(e_{0}, e_{1}\right) \rightarrow 0
$$

and the tautological sequence on $G(A, 2)$ becomes

$$
0 \rightarrow \mathscr{L}\left(\left(e_{2}, \ldots, e_{a}\right)\right) \rightarrow \mathscr{O}_{G} \otimes A \rightarrow \mathscr{L}\left(\left(e_{0}, e_{1}\right)\right) \rightarrow 0 .
$$

Since $\left(e_{0}, e_{1}\right)=\operatorname{ind}_{B}^{P} \epsilon_{0}$ as $P$-modules we get

$$
S^{a} \mathscr{E}=S^{a} \mathscr{L}\left(\left(e_{0}, e_{1}\right)\right)=\mathscr{L}\left(S^{a}\left(e_{0}, e_{1}\right)\right)=\mathscr{L}\left(\operatorname{ind}_{B}^{P}\left(a \epsilon_{0}\right)\right)
$$

and

$$
\mathscr{L}:=\left(S^{a \mathscr{E}}\right)(-2) \otimes \wedge^{a+1} A=\mathscr{L}\left(\operatorname{ind}_{B}^{P}\left((a-1) \epsilon_{0}-\epsilon_{1}+\sum_{2}^{a} \epsilon_{i}\right)\right) .
$$

By the Borel-Bott-Weil theorem we find that

$$
H^{a-1} \mathscr{L}(-a+1)=H^{a-1}\left(-a \epsilon_{1}+\sum_{2}^{a} \epsilon_{i}\right)=H^{0}\left(-\epsilon_{a}\right)=A^{*}
$$

and

$$
H^{a-1} \mathscr{L}(-a+2)=H^{a-1}\left(\epsilon_{0}-(a-1) \epsilon_{1}+\sum_{2}^{a} \epsilon_{i}\right)=H^{0}\left(\epsilon_{0}\right)=A .
$$

Since we find $H^{i} \mathscr{L}(-i)=0$ and $H^{i} \mathscr{L}(-i+1)=0$ for $i \neq a-1$, part a. follows.

We find that

$$
\begin{aligned}
H^{0} \mathscr{L}(p) & =H^{0}\left((a+p-1) \epsilon_{0}+(p-1) \epsilon_{1}+\sum_{2}^{a} \epsilon_{i}\right) \\
& =S_{a+p-2, p-2} A \otimes \wedge^{a+1} A, \quad p \geq 2
\end{aligned}
$$

and by Borel-Bott-Weil that

$$
\begin{aligned}
H^{2 a-2} \mathscr{L}(2-2 a-r) & =\wedge^{a+1} A \otimes H^{2 a-2}\left((-a-r) \epsilon_{0}+(-2 a-r) \epsilon_{1}\right) \\
& =\wedge^{a+1} A \otimes\left(\wedge^{a+1} A^{*}\right)^{\otimes 2} \otimes S_{a+r-1, r-1} A^{*} \\
& =S_{a+r-1, r-1} A^{*} \otimes \wedge^{a+1} A^{*}, \quad p=-r<0
\end{aligned}
$$


and also that $H^{i} \mathscr{L}(p-i)=0$ for all other $i$ and $p$. Hence b. follows.

\section{Some further examples}

Example 6.1. Consider the map

$$
\omega_{E}(1) \otimes V \stackrel{\phi}{\longrightarrow} \omega_{E}(-1) \otimes V^{*}
$$

corresponding to the map $\wedge^{2} V^{*} \otimes V \rightarrow V^{*}$. Here we do not get associated a coherent sheaf to the Tate resolution $T(\phi)$ but instead a complex, the truncated Koszul complex

$$
\mathscr{O}_{\mathrm{P}(W)}(-v) \otimes \wedge^{v} W \rightarrow \cdots \rightarrow \mathscr{O}_{\mathrm{P}(W)}(-2) \otimes \wedge^{2} W \rightarrow \mathscr{O}_{\mathrm{P}(W)}(-1) \otimes W
$$

In fact, let $M$ be $\oplus_{i=1}^{v} \wedge^{i} W$ which is a subquotient of $\omega_{E}$. Then we see that (5) is $L(M)^{\sim}$.

Since $\omega_{E}(1) \otimes V$ is the projective cover of $M$ and $\omega_{E}(-1) \otimes W$ is the injective hull, we get $M=\operatorname{Im} \phi$ and so $T(\phi)$ corresponds to (5).

EXAMPLE 6.2. Suppose $v$ is odd. Let $\wedge^{2} W \rightarrow k$ be a non-degenerate symplectic form. Then the map $\wedge^{v-1} W \rightarrow k$ gives a morphism

$$
\omega_{E}(v-1) \stackrel{\phi}{\longrightarrow} \omega_{E}
$$

equivariant under the symplectic group $\operatorname{Sp}(W)$. Since the image of $\phi$ is $k \oplus$ $W \oplus \operatorname{Im} \phi_{2}, T(\phi)$ corresponds to a null correlation bundle on $\mathrm{P}(W)$ (which is $\mathrm{Sp}(W)$-equivariant).

\section{REFERENCES}

1. Bernstein, I., Gelfand, I., Gelfand, S., Algebraic bundles over $\mathbf{P}^{r}$ and problems of linear algebra, Funktsional. Anal. i Prilozhen. 12 (1978), English translation in Funct. Anal. Appl. 12 (1978), 212-214.

2. Eisenbud, D., Commutative Algebra, Grad. Texts in Math. 150 (1995).

3. Eisenbud, D., Fløystad, G., Schreyer, F.-O., Sheaf Cohomology and Free Resolutions over Exterior Algebras, Trans. Amer. Math. Soc. 355 (2003), 4397-4426.

4. Fløystad, G., Describing coherent sheaves on projective spaces via Koszul duality, http://arXiv.org/abs/math.AG/0012263.

5. Jantzen, J. C., Representations of Algebraic Groups, Pure Appl. Math. 131 (1987).

MATEMATISK INSTITUTT

JOHS. BRUNSGT. 12

5008 BERGEN

NORWAY

E-mail: gunnar@mi.uib.no 